\title{
Paradoxical Brain Embolism with Klippel-Trenaunay Syndrome
}

\author{
Kenichiro Sakai, Kensaku Sibazaki, Kazumi Kimura, Kazuto Kobayashi, \\ Noriko Matsumoto and Yasuyuki Iguchi
}

\begin{abstract}
Cerebrovascular diseases in patients with Klippel-Trenaunay Syndrome (KTS) are uncommon, and the mechanism of stroke has remained elusive. We describe a patient with KTS who experienced a transient ischemic attack (TIA). Contrast-transcranial Doppler with the Valsalva maneuver revealed a right-to-left shunt and contrast-transesophageal echocardiography confirmed patent foramen ovale. Ultrasonography revealed dilated superficial and deep veins in the lower extremities; the D-dimer level was high and indicated hypercoagulability. Therefore, the mechanism of TIA was diagnosed as paradoxical embolism. To the best of our knowledge, this is the first case report of paradoxical embolism in a patient with KTS.
\end{abstract}

Key words: Klippel-Trenaunay syndrome, patent foramen ovale, paradoxical embolism

(Intern Med 50: 141-143, 2011)

(DOI: 10.2169/internalmedicine.50.3870)

\section{Introduction}

Klippel-Trenaunay syndrome (KTS) is a rare congenital anomaly characterized by capillary malformations (port-wine stains), one hypertrophied leg, and large varicose veins on the lateral aspect of the leg (1). Few reports have been published about ischemic stroke associated with KTS and the cause of brain ischemic events has remained unknown $(2,3)$. Contrast-transcranial Doppler (c-TCD) and contrast-transesophageal echocardiography (c-TEE) are useful tools with which to detect a right-to-left shunt such as patent foramen ovale (PFO) and pulmonary arteriovenous fistula (4). We herein describe a transient ischemic attack (TIA) in a patient with KTS in which c-TCD revealed a right-to-left shunt and c-TEE confirmed the presence of PFO. The proposed mechanism of TIA was paradoxical embolism.

\section{Case Report}

A 31-year-old Canadian man had taken warfarin and aspirin because of functional failure of veins in both legs 5 years previously. $\mathrm{He}$ did not have a history of migraine. He was admitted to our hospital due to sudden onset of dysarthria and numbness on the right side of his body while sitting at rest after dinner.

An examination at the time of admission showed the following: height, $183 \mathrm{~cm}$; weight, $70 \mathrm{~kg}$; blood pressure, 110/ $60 \mathrm{mmHg}$; pulse rate, $66 \mathrm{bpm}$ with regular sinus rhythm; temperature, $36.4^{\circ} \mathrm{C}$. Heart and respiratory sounds were clear. No neck or orbital bruits were evident. Varicose veins and port-wine stains were found on bilateral upper and lower limbs (Fig. 1), indicating a diagnosis of KTS. Neurological findings were normal except for hyperesthesia on the right side of his face and on a finger of his right hand.

Laboratory examinations upon admission revealed the following: D-dimer, $11.3 \mu \mathrm{g} / \mathrm{mL}$ (normal, <1.0); thrombinantithrombin III complex, $9.9 \mathrm{ng} / \mathrm{mL}$ (normal, <3.0) and a prothrombin time - international normalized ratio (PT-INR) of 1.37 (normal, <1.2).

On hospital day 1 , the patient was examined using a 1.5T MRI unit (Signa EchoSpeed Horizon; GE Medical Systems, Milwaukee, WI). Diffusion-weighted magnetic resonance imaging (DWI) and T2-weighted imaging showed no hyper-intense lesions. Magnetic resonance angiography and neck ultrasonography demonstrated no atherosclerotic changes in cerebral vessels. Electrocardiography (Holter 24

Department of Stroke Medicine, Kawasaki Medical School, Japan

Received for publication April 26, 2010; Accepted for publication August 25, 2010

Correspondence to Dr. Kenichiro Sakai, k-sakai@med.kawasaki-m.ac.jp 

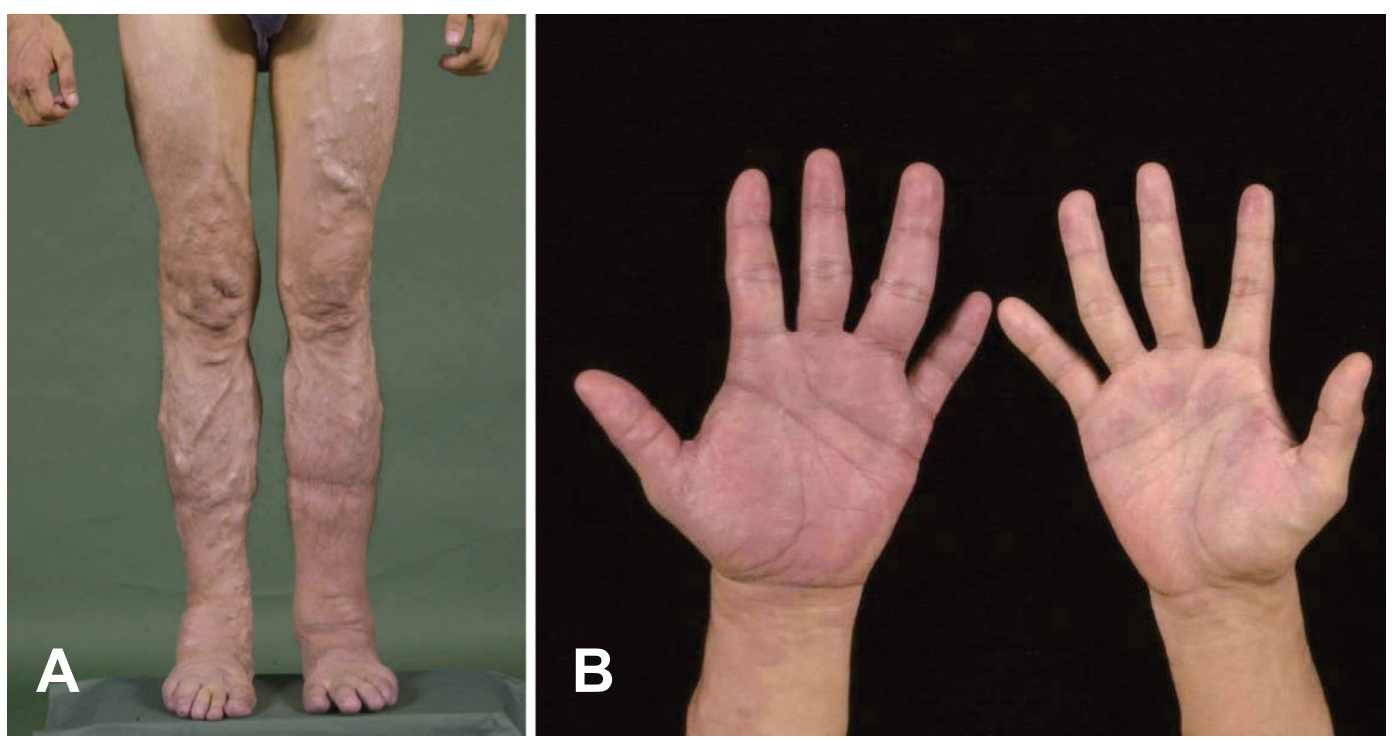

Figure 1. Patient features. A: Varicose veins on his legs; B: Port-wine stain on his hands.

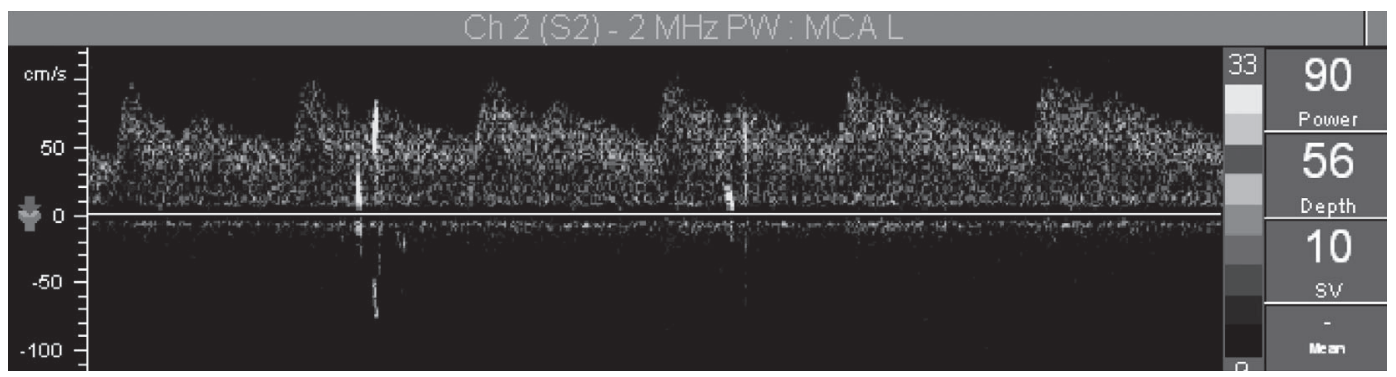

Figure 2. Findings of transcranial Doppler examination using saline contrast medium. Contrast transcranial Doppler (TCD) demonstrates evident right-to-left shunt.

hours electrocardiography) showed a normal sinus rhythm without atrial fibrillation. The possibility of a right-to-left shunt was examined using TCD with saline contrast medium as follows. Saline $(9 \mathrm{~mL})$ and air $(1 \mathrm{~mL})$ were agitated between two $10-\mathrm{mL}$ syringes connected by a three-way stopcock (4). Contrast-TCD showed multiple high intensity signals from the left MCA with the Valsalva maneuver (Fig. 2). The results confirmed a right-to-left shunt and c-TEE findings confirmed patent foramen ovale (PFO). Ultrasonography of the lower limb showed obviously enlarged superficial and deep veins, but deep venous thrombosis (DVT) was undetectable. Warfarin was administered to maintain the PTINR between 2 and 3 . The neurological findings disappeared within 1 hour after onset.

Follow-up MRI two weeks after admission demonstrated two hyper-intense lesions at the left cerebellum hemisphere on DWI and T2-weighted MRI. We suspected the lesion to be an asymptomatic infarct because he did not have ataxia or vertigo during the two weeks after the TIA attack.

\section{Discussion}

We described a patient with KTS who experienced a TIA. Little is understood about stroke associated with KTS. Among 104 children with stroke investigated by Salih et al only one had KTS (2). Brunaud et al described a 39-yearold man with KTS who experienced an ischemic attack due to internal carotid artery dissection (3). To the best of our knowledge, this is the first case report to describe paradoxical embolism as the cause of a brain ischemic event in a patient with KTS.

About $30 \%$ of the general population has PFO (5). Paradoxical embolism through PFO is a frequent mechanism of ischemic stroke, especially among pediatric and younger patients. Therefore, we initially suspected that the mechanism of TIA in our patient was paradoxical embolism.

Both C-TCD and c-TEE can detect a right-to-left shunt. In the present patient, c-TCD detected a right-to-left shunt and TEE confirmed the presence of PFO. All of the following criteria must be met to establish a diagnosis of paradoxical embolism: embolic stroke, a right-to-left shunt and an embolic source such as DVT or pulmonary embolism (PE) (6). Our patient did not have DVT or PE. However, superficial and deep veins in his lower extremities were dilated, and the D-dimer level was high, indicating hypercoagulability (7). Therefore, we suspected DVT.

Both DVT and PE are known complications of KTS. Baskerville et al reported a $17 \%$ and $22 \%$ incidence of DVT and PE, respectively, in patients with KTS (8). Therefore, a patient with KTS with a right-to-left shunt, such as that 
caused by PFO and pulmonary arteriovenous fistula, is likely to have paradoxical embolism. Although it is unclear whether or not patients with KTS tend to have PFO, c-TCD should be performed. If a right-to-left shunt is revealed, then the patient should undergo intensive care such as the administration of antiplatelet agents and anticoagulants, surgical closure or the application of percutaneous closure devices to prevent paradoxical embolism.

In conclusion, we described a patient with KTS who experienced TIA. Conditions such as DVT and PE are frequent complications in patients with KTS. A right-to-left shunt in a patient with KTS indicates probable development of paradoxical embolism.

The authors state that they have no Conflict of Interest (COI).

\section{References}

1. Delis KT, Gloviczki P, Wennberg PW, Rooke TW, Driscoll DJ. Hemodynamic impairment, venous segmental disease, and clinical severity scoring in limbs with Klippel-Trenaunay syndrome. J Vasc Surg 45: 561-567, 2007.

2. Salih MA, Murshid WR, Zahraa JN, et al. Congenital and genetic cerebrovascular anomalies as risk factors for stroke in Saudi children. Saudi Med J 27 Suppl 1 : S53-S60, 2006.

3. Brunaud V, Delerue O, Muller JP, Destee A. Klippel-Trenaunay syndrome and ischemic neurologic complications. Rev Neurol (Paris) 150: 50-54, 1994 (in French).

4. Kimura K, Minematsu K, Wada K, et al. Transcranial Doppler of a paradoxical brain embolism associated with a pulmonary arteriovenous fistula. AJNR Am J Neuroradiol 20: 1881-1884, 1999.

5. de Belder MA, Tourikis L, Leech G, Camm AJ. Risk of patent foramen ovale for thromboembolic events in all age groups. Am J Cardiol 69: 1316-1320, 1992.

6. Yasaka M, Otsubo R, Oe H, Minematsu K. Is stroke a paradoxical embolism in patients with patent foramen ovale? Intern Med 44: 434-438, 2005

7. Kelly J, Rudd A, Lewis R, Hunt BJ. Venous thromboembolism after acute stroke. Stroke 32: 262-267, 2001.

8. Baskerville PA, Ackroyd JS, Lea Thomas M, Browse NL. The Klippel-Trenaunay syndrome: clinical, radiological and haemodynamic features and management. Br J Surg 72: 232-236, 1985.

(C) 2011 The Japanese Society of Internal Medicine http://www.naika.or.jp/imindex.html 